Journal of Inequalities and Special Functions

ISSN: 2217-4303, URL: WWW.ILIRIAS.COM/JIASF

Volume 12 Issue 4 (2021), Pages 1-15.

DOI:10.54379/JIASF-2021-4-1

\title{
GENERALIZED MULTIVARIATE PRABHAKAR TYPE FRACTIONAL INTEGRALS AND INEQUALITIES
}

\author{
GEORGE A. ANASTASSIOU
}

\begin{abstract}
We introduce here the mixed generalized multivariate Prabhakar type left and right fractional integrals and study their basic properties, such as preservation of continuity and their boundedness as positive linear operators. Then we produce an interesting variety of related multivariate left and right fractional Hardy type inequalities under convexity. We introduce also other related multivariate fractional integrals.
\end{abstract}

\section{BACKGROUND}

This work is inspired by [6], [8, [9, [1] - [15].

Here we consider the Prabhakar function (also known as the three parameter Mittag-Leffler function, an entire function if $z \in \mathbb{C}$ ), (see [5], p. 97; [4])

$$
E_{\alpha, \beta}^{\gamma}(z)=\sum_{k=0}^{\infty} \frac{(\gamma)_{k}}{k ! \Gamma(\alpha k+\beta)} z^{k}
$$

where $\Gamma$ is the gamma function; $\alpha, \beta, \gamma \in \mathbb{R}: \alpha, \beta>0, z \in \mathbb{R}$, and $(\gamma)_{k}=$ $\gamma(\gamma+1) \ldots(\gamma+k-1)$. It is $E_{\alpha, \beta}^{0}(z)=\frac{1}{\Gamma(\beta)}$.

Let $a, b \in \mathbb{R}, a<b$ and $x \in[a, b] ; f \in C([a, b])$. Let also $\psi \in C^{1}([a, b])$ which is increasing. In [3] we defined and studied the left and right Prabhakar fractional integrals with respect to $\psi$ as follows:

$$
\left(e_{\rho, \mu, \omega, a+}^{\gamma ; \psi} f\right)(x)=\int_{a}^{x} \psi^{\prime}(t)(\psi(x)-\psi(t))^{\mu-1} E_{\rho, \mu}^{\gamma}\left[\omega(\psi(x)-\psi(t))^{\rho}\right] f(t) d t,
$$

and

$$
\left(e_{\rho, \mu, \omega, b-}^{\gamma ; \psi} f\right)(x)=\int_{x}^{b} \psi^{\prime}(t)(\psi(t)-\psi(x))^{\mu-1} E_{\rho, \mu}^{\gamma}\left[\omega(\psi(t)-\psi(x))^{\rho}\right] f(t) d t,
$$

where $\rho, \mu>0 ; \gamma, \omega \in \mathbb{R}$, which are continuous functions $\underline{3}$.

In this work we define and study the multivariate analogs of (2) and (3).

2000 Mathematics Subject Classification. 26A33, 26D10, $26 \mathrm{D} 15$.

Key words and phrases. Prabhakar fractional integral, Hardy inequality, generalized fractional integral, convexity.

(C)2021 Ilirias Research Institute, Prishtinë, Kosovë.

Submitted May 15, 2021. Published October 24, 2021.

Communicated by M. Bohner. 
Let $\prod_{i=1}^{N}\left[a_{i}, b_{i}\right] \subset \mathbb{R}^{N}, N>1, a_{i}<b_{i}, a_{i}, b_{i} \in \mathbb{R} ; f \in C\left(\prod_{i=1}^{N}\left[a_{i}, b_{i}\right]\right)$. Let also $x_{i}, t_{i} \in\left[a_{i}, b_{i}\right] ; \rho_{i}, \mu_{i}>0 ; \gamma_{i}, \omega_{i} \in \mathbb{R} ; i=1, \ldots, N$. Here $\psi_{i} \in C^{1}\left(\left[a_{i}, b_{i}\right]\right)$ which is increasing, $i=1, \ldots, N$. We set $a=\left(a_{1}, \ldots, a_{N}\right), b=\left(b_{1}, \ldots, b_{N}\right), x=$ $\left(x_{1}, \ldots, x_{N}\right), t=\left(t_{1}, \ldots, t_{N}\right) ; \rho=\left(\rho_{1}, \ldots, \rho_{N}\right), \mu=\left(\mu_{1}, \ldots, \mu_{N}\right), \gamma=\left(\gamma_{1}, \ldots, \gamma_{N}\right)$, $\omega=\left(\omega_{1}, \ldots, \omega_{N}\right), \psi:=\left(\psi_{1}, \ldots, \psi_{N}\right)$.

We define the left and right mixed Prabhakar multiple fractional integrals with respect to $\psi$, respectively, as follows:

$$
\begin{gathered}
\left({ }^{M} e_{\rho, \mu, \omega, a+}^{\gamma ; \psi} f\right)(x):=\int_{a_{1}}^{x_{1}} \ldots \int_{a_{N}}^{x_{N}} \prod_{i=1}^{N}\left[\psi_{i}^{\prime}\left(t_{i}\right)\left(\psi_{i}\left(x_{i}\right)-\psi_{i}\left(t_{i}\right)\right)^{\mu_{i}-1}\right. \\
\left.E_{\rho_{i}, \mu_{i}}^{\gamma_{i}}\left[\omega_{i}\left(\psi_{i}\left(x_{i}\right)-\psi_{i}\left(t_{i}\right)\right)^{\rho_{i}}\right]\right] f\left(t_{1}, \ldots, t_{N}\right) d t_{1} \ldots d t_{N},
\end{gathered}
$$

with $x_{i} \geq a_{i}, i=1, \ldots, N$;

$$
\begin{gathered}
\left({ }^{M} e_{\rho, \mu, \omega, b-}^{\gamma ; \psi} f\right)(x):=\int_{x_{1}}^{b_{1}} \ldots \int_{x_{N}}^{b_{N}} \prod_{i=1}^{N}\left[\psi_{i}^{\prime}\left(t_{i}\right)\left(\psi_{i}\left(t_{i}\right)-\psi_{i}\left(x_{i}\right)\right)^{\mu_{i}-1}\right. \\
\left.E_{\rho_{i}, \mu_{i}}^{\gamma_{i}}\left[\omega_{i}\left(\psi_{i}\left(t_{i}\right)-\psi_{i}\left(x_{i}\right)\right)^{\rho_{i}}\right]\right] f\left(t_{1}, \ldots, t_{N}\right) d t_{1} \ldots d t_{N}
\end{gathered}
$$

with $x_{i} \leq b_{i}, i=1, \ldots, N$.

We give

Theorem 1.1. Let $\rho_{i}, \gamma_{i}>0, \mu_{i} \geq 1, \omega_{i} \in \mathbb{R} ; i=1, \ldots, N$. Then $\left({ }^{M} e_{\rho, \mu, \omega, a+}^{\gamma ; \psi} f\right)$, $\left({ }^{M} e_{\rho, \mu, \omega, b-}^{\gamma ; \psi} f\right) \in C\left(\prod_{i=1}^{N}\left[a_{i}, b_{i}\right]\right)$.

Proof. It is enough to prove that $\left({ }^{M} e_{\rho, \mu, \omega, a+}^{\gamma ; \psi} f\right) \in C\left(\prod_{i=1}^{N}\left[a_{i}, b_{i}\right]\right)$. The proof for the second is similar and omitted.

One can write

$$
\begin{gathered}
\left({ }^{M} e_{\rho, \mu, \omega, a+}^{\gamma ; \psi} f\right)\left(x_{0}\right)=\int_{\prod_{i=1}^{N}\left[a_{i}, b_{i}\right]} \chi_{\prod_{i=1}^{N}\left[a_{i}, x_{0 i}\right]}(t) \prod_{i=1}^{N}\left[\psi_{i}^{\prime}\left(t_{i}\right)\left(\psi_{i}\left(x_{0 i}\right)-\psi_{i}\left(t_{i}\right)\right)^{\mu_{i}-1}\right. \\
\left.E_{\rho_{i}, \mu_{i}}^{\gamma_{i}}\left[\omega_{i}\left(\psi_{i}\left(x_{0 i}\right)-\psi_{i}\left(t_{i}\right)\right)^{\rho_{i}}\right]\right] f(t) d t
\end{gathered}
$$

where $\chi$ is the characteristic function and $x_{0}=\left(x_{01}, \ldots, x_{0 N}\right) \in \prod_{i=1}^{N}\left[a_{i}, b_{i}\right]$.

Let $x_{m} \rightarrow x_{0}$ as $m \rightarrow+\infty$, where $x_{m} \in \prod_{i=1}^{N}\left[a_{i}, b_{i}\right]$; with $x_{m}=\left(x_{m 1}, \ldots, x_{m N}\right)$.

We will prove that $\left({ }^{M} e_{\rho, \mu, \omega, a+}^{\gamma ; \psi} f\right)\left(x_{m}\right) \rightarrow\left({ }^{M} e_{\rho, \mu, \omega, a+}^{\gamma ; \psi} f\right)\left(x_{0}\right)$, as $m \rightarrow+\infty$.

We have that

$$
\begin{aligned}
\left({ }^{M} e_{\rho, \mu, \omega, a+}^{\gamma ; \psi} f\right)\left(x_{m}\right) & =\int_{\prod_{i=1}^{N}\left[a_{i}, b_{i}\right]} \chi_{\prod_{i=1}^{N}\left[a_{i}, x_{m i}\right]}(t) \prod_{i=1}^{N}\left[\psi_{i}^{\prime}\left(t_{i}\right)\left(\psi_{i}\left(x_{m i}\right)-\psi_{i}\left(t_{i}\right)\right)^{\mu_{i}-1}\right. \\
& \left.E_{\rho_{i}, \mu_{i}}^{\gamma_{i}}\left[\omega_{i}\left(\psi_{i}\left(x_{m i}\right)-\psi_{i}\left(t_{i}\right)\right)^{\rho_{i}}\right]\right] f(t) d t
\end{aligned}
$$


We notice that $\chi_{\prod_{i=1}^{N}\left[a_{i}, x_{m i}\right]}(t) \rightarrow \chi_{\prod_{i=1}^{N}\left[a_{i}, x_{0 i}\right]}(t)$, a.e.; also it holds

$$
\begin{aligned}
& T_{t}\left(x_{m}\right):=\prod_{i=1}^{N}\left[\psi_{i}^{\prime}\left(t_{i}\right)\left(\psi_{i}\left(x_{m i}\right)-\psi_{i}\left(t_{i}\right)\right)^{\mu_{i}-1} E_{\rho_{i}, \mu_{i}}^{\gamma_{i}}\left[\omega_{i}\left(\psi_{i}\left(x_{m i}\right)-\psi_{i}\left(t_{i}\right)\right)^{\rho_{i}}\right]\right] \\
& \rightarrow \prod_{i=1}^{N}\left[\psi_{i}^{\prime}\left(t_{i}\right)\left(\psi_{i}\left(x_{0 i}\right)-\psi_{i}\left(t_{i}\right)\right)^{\mu_{i}-1} E_{\rho_{i}, \mu_{i}}^{\gamma_{i}}\left[\omega_{i}\left(\psi_{i}\left(x_{0 i}\right)-\psi_{i}\left(t_{i}\right)\right)^{\rho_{i}}\right]\right]=: T_{t}\left(x_{0}\right),
\end{aligned}
$$

as $m \rightarrow+\infty$.

Furthermore we obtain

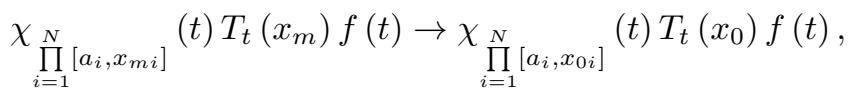

a.e. on $\prod_{i=1}^{N}\left[a_{i}, b_{i}\right]$, as $m \rightarrow+\infty$.

However, we have that

$$
\begin{gathered}
\chi_{\prod_{i=1}^{N}\left[a_{i}, x_{m i}\right]}(t)\left|T_{t}\left(x_{m}\right)\right||f(t)| \leq\left|T_{t}\left(x_{m}\right)\right||f(t)| \leq \\
\prod_{i=1}^{N}\left[\left\|\psi_{i}^{\prime}\right\|_{\infty,\left[a_{i}, b_{i}\right]}\left(\psi_{i}\left(b_{i}\right)-\psi_{i}\left(a_{i}\right)\right)^{\mu_{i}-1} E_{\rho_{i}, \mu_{i}}^{\gamma_{i}}\left[\left|\omega_{i}\right|\left(\psi_{i}\left(b_{i}\right)-\psi_{i}\left(a_{i}\right)\right)^{\left.\rho_{i}\right]}\right]\right. \\
\|f\|_{\infty, \prod_{i=1}^{N}\left[a_{i}, b_{i}\right]}<+\infty .
\end{gathered}
$$

Thus, by the dominated convergence theorem, we derive

$$
\int_{\prod_{i=1}^{N}\left[a_{i}, b_{i}\right]} \chi_{\prod_{i=1}^{N}\left[a_{i}, x_{m i}\right]}(t) T_{t}\left(x_{m}\right) f(t) d t \rightarrow \int_{\prod_{i=1}^{N}\left[a_{i}, b_{i}\right]} \chi_{\prod_{i=1}^{N}\left[a_{i}, x_{0 i}\right]}(t) T_{t}\left(x_{0}\right) f(t) d t
$$

as $m \rightarrow+\infty$, proving the claim.

Conjecture 1.2. Functions (4) and (5) must be continuous when $\rho_{i}, \mu_{i}>0$; $\gamma_{i}, \omega_{i} \in \mathbb{R} ; i=1, \ldots, N$.

We also present the following basic Hardy type inequalities.

Theorem 1.3. Let $\rho_{i}, \mu_{i}, \gamma_{i}, \omega_{i}>0, i=1, \ldots, N$. Then

$$
\begin{gathered}
\left\{\left\|\left({ }^{M} e_{\rho, \mu, \omega, a+}^{\gamma ; \psi} f\right)\right\|_{\infty, \prod_{i=1}^{N}\left[a_{i}, b_{i}\right]},\left\|\left({ }^{M} e_{\rho, \mu, \omega, b-}^{\gamma ; \psi} f\right)\right\|_{\infty, \prod_{i=1}^{N}\left[a_{i}, b_{i}\right]}\right\} \leq \\
\left\{\left\|\left({ }^{M} e_{\rho, \mu, \omega, a+}^{\gamma ; \psi}|f|\right)\right\|_{\infty, \prod_{i=1}^{N}\left[a_{i}, b_{i}\right]},\left\|\left({ }^{M} e_{\rho, \mu, \omega, b-}^{\gamma ; \psi}|f|\right)\right\|_{\infty, \prod_{i=1}^{N}\left[a_{i}, b_{i}\right]}\right\} \leq \\
{\left[\prod_{i=1}^{N}\left[\left(\psi_{i}\left(b_{i}\right)-\psi_{i}\left(a_{i}\right)\right)^{\mu_{i}} E_{\rho_{i}, \mu_{i}+1}^{\gamma_{i}}\left[\omega_{i}\left(\psi_{i}\left(b_{i}\right)-\psi_{i}\left(a_{i}\right)\right)^{\rho_{i}}\right]\right]\right]\|f\|_{\infty, \prod_{i=1}^{N}\left[a_{i}, b_{i}\right]}<\infty,}
\end{gathered}
$$

respectively.

Here (4) and (5) are bounded and positive linear operators. 
Proof. It is enough to estimate only $\left\|\left({ }^{M} e_{\rho, \mu, \omega, a+}^{\gamma ; \psi} f\right)\right\|_{\infty, \prod_{i=1}^{N}\left[a_{i}, b_{i}\right]}$. The proof of the second as similar is omitted. We have that

$$
\begin{aligned}
& \left|\left({ }^{M} e_{\rho, \mu, \omega, a+}^{\gamma ; \psi} f\right)(x)\right| \stackrel{\text { 嵖 }}{\leq}\left({ }^{M} e_{\rho, \mu, \omega, a+}^{\gamma ; \psi}|f|\right)(x)= \\
& \int_{a_{1}}^{x_{1}} \ldots \int_{a_{N}}^{x_{N}} \prod_{i=1}^{N}\left[\psi_{i}^{\prime}\left(t_{i}\right)\left(\psi_{i}\left(x_{i}\right)-\psi_{i}\left(t_{i}\right)\right)^{\mu_{i}-1}\right. \\
& \left.E_{\rho_{i}, \mu_{i}}^{\gamma_{i}}\left[\omega_{i}\left(\psi_{i}\left(x_{i}\right)-\psi_{i}\left(t_{i}\right)\right)^{\rho_{i}}\right]\right]\left|f\left(t_{1}, \ldots, t_{N}\right)\right| d t_{1} \ldots d t_{N} \leq \\
& \|f\|_{\infty, \prod_{i=1}^{N}\left[a_{i}, b_{i}\right]} \prod_{i=1}^{N}\left(\int_{a_{i}}^{x_{i}} \psi_{i}^{\prime}\left(t_{i}\right)\left(\psi_{i}\left(x_{i}\right)-\psi_{i}\left(t_{i}\right)\right)^{\mu_{i}-1}\right. \\
& \left.E_{\rho_{i}, \mu_{i}}^{\gamma_{i}}\left[\omega_{i}\left(\psi_{i}\left(x_{i}\right)-\psi_{i}\left(t_{i}\right)\right)^{\rho_{i}}\right] d t_{i}\right)= \\
& \|f\|_{\infty, \prod_{i=1}^{N}\left[a_{i}, b_{i}\right]} \prod_{i=1}^{N}\left(\int_{a_{i}}^{x_{i}} \psi_{i}^{\prime}\left(t_{i}\right)\left(\psi_{i}\left(x_{i}\right)-\psi_{i}\left(t_{i}\right)\right)^{\mu_{i}-1}\right.
\end{aligned}
$$

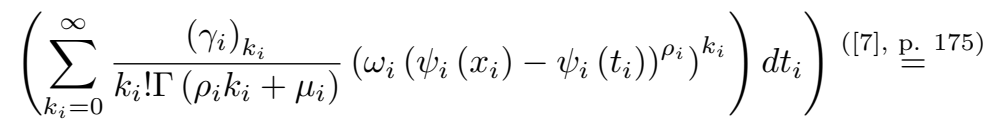

$$
\begin{aligned}
& \|f\|_{\infty, \prod_{i=1}^{N}\left[a_{i}, b_{i}\right]} \prod_{i=1}^{N}\left[\sum_{k_{i}=0}^{\infty} \frac{\left(\gamma_{i}\right)_{k_{i}} \omega_{i}^{k_{i}}}{k_{i} ! \Gamma\left(\rho_{i} k_{i}+\mu_{i}\right)} \int_{a_{i}}^{x_{i}} \psi_{i}^{\prime}\left(t_{i}\right)\left(\psi_{i}\left(x_{i}\right)-\psi_{i}\left(t_{i}\right)\right)^{\left(\rho_{i} k_{i}+\mu_{i}\right)-1} d t_{i}\right]= \\
& \|f\|_{\infty, \prod_{i=1}^{N}\left[a_{i}, b_{i}\right]} \prod_{i=1}^{N}\left[\sum_{k_{i}=0}^{\infty} \frac{\left(\gamma_{i}\right)_{k_{i}} \omega_{i}^{k_{i}}}{k_{i} ! \Gamma\left(\rho_{i} k_{i}+\mu_{i}+1\right)}\left(\psi_{i}\left(x_{i}\right)-\psi_{i}\left(a_{i}\right)\right)^{\rho_{i} k_{i}+\mu_{i}}\right]= \\
& \|f\|_{\infty, \prod_{i=1}^{N}\left[a_{i}, b_{i}\right]} \prod_{i=1}^{N}\left[\left(\psi_{i}\left(x_{i}\right)-\psi_{i}\left(a_{i}\right)\right)^{\mu_{i}} \sum_{k_{i}=0}^{\infty} \frac{\left(\gamma_{i}\right)_{k_{i}}\left(\omega_{i}\left(\psi_{i}\left(x_{i}\right)-\psi_{i}\left(a_{i}\right)\right)^{\rho_{i}}\right)^{k_{i}}}{k_{i} ! \Gamma\left(\rho_{i} k_{i}+\mu_{i}+1\right)}\right]= \\
& \|f\|_{\infty, \prod_{i=1}^{N}\left[a_{i}, b_{i}\right]} \prod_{i=1}^{N}\left[\left(\psi_{i}\left(x_{i}\right)-\psi_{i}\left(a_{i}\right)\right)^{\mu_{i}} E_{\rho_{i}, \mu_{i}+1}^{\gamma_{i}}\left[\omega_{i}\left(\psi_{i}\left(x_{i}\right)-\psi_{i}\left(a_{i}\right)\right)^{\rho_{i}}\right]\right] \leq \\
& \|f\|_{\infty, \prod_{i=1}^{N}\left[a_{i}, b_{i}\right]} \prod_{i=1}^{N}\left[\left(\psi_{i}\left(b_{i}\right)-\psi_{i}\left(a_{i}\right)\right)^{\mu_{i}} E_{\rho_{i}, \mu_{i}+1}^{\gamma_{i}}\left[\omega_{i}\left(\psi_{i}\left(b_{i}\right)-\psi_{i}\left(a_{i}\right)\right)^{\rho_{i}}\right]\right], \\
& \forall x \in \prod_{i=1}^{N}\left[a_{i}, b_{i}\right] \text {. }
\end{aligned}
$$

The claim is proved.

We make

Remark 1.4. Let $\rho_{i}, \mu_{i}, \gamma_{i}, \omega_{i}>0, i=1, \ldots, N$. We define the kernels $(y=$ $\left.\left(y_{1}, \ldots, y_{N}\right) \in \prod_{i=1}^{N}\left(a_{i}, b_{i}\right)\right)$

$$
\begin{gathered}
k_{a+}(x, y)=\chi_{\prod_{i=1}^{N}\left(a_{i}, x_{i}\right]}(y) \prod_{i=1}^{N}\left[\psi_{i}^{\prime}\left(y_{i}\right)\left(\psi_{i}\left(x_{i}\right)-\psi_{i}\left(y_{i}\right)\right)^{\mu_{i}-1}\right. \\
\left.E_{\rho_{i}, \mu_{i}}^{\gamma_{i}}\left[\omega_{i}\left(\psi_{i}\left(x_{i}\right)-\psi_{i}\left(y_{i}\right)\right)^{\rho_{i}}\right]\right]
\end{gathered}
$$


and

$$
\begin{gathered}
k_{b-}(x, y)=\chi_{\prod_{i=1}^{N}\left[x_{i}, b_{i}\right)}(y) \prod_{i=1}^{N}\left[\psi_{i}^{\prime}\left(y_{i}\right)\left(\psi_{i}\left(y_{i}\right)-\psi_{i}\left(x_{i}\right)\right)^{\mu_{i}-1}\right. \\
\left.E_{\rho_{i}, \mu_{i}}^{\gamma_{i}}\left[\omega_{i}\left(\psi_{i}\left(y_{i}\right)-\psi_{i}\left(x_{i}\right)\right)^{\rho_{i}}\right]\right],
\end{gathered}
$$

$\forall x, y \in \prod_{i=1}^{N}\left(a_{i}, b_{i}\right)$.

We compute the integral

$$
\begin{gathered}
K_{a+}(x):=\int_{\prod_{i=1}^{N}\left(a_{i}, b_{i}\right)} k_{a+}(x, y) d y \stackrel{\text { 幽 }}{=}\left({ }^{M} e_{\rho, \mu, \omega, a+}^{\gamma ; \psi} 1\right)(x)= \\
\int_{a_{1}}^{x_{1}} \ldots \int_{a_{N}}^{x_{N}} \prod_{i=1}^{N}\left[\psi_{i}^{\prime}\left(t_{i}\right)\left(\psi_{i}\left(x_{i}\right)-\psi_{i}\left(t_{i}\right)\right)^{\mu_{i}-1}\right. \\
\left.E_{\rho_{i}, \mu_{i}}^{\gamma_{i}}\left[\omega_{i}\left(\psi_{i}\left(x_{i}\right)-\psi_{i}\left(t_{i}\right)\right)^{\rho_{i}}\right]\right] d t_{1} \ldots d t_{N}= \\
\prod_{i=1}^{N}\left(\int_{a_{i}}^{x_{i}} \psi_{i}^{\prime}\left(t_{i}\right)\left(\psi_{i}\left(x_{i}\right)-\psi_{i}\left(t_{i}\right)\right)^{\mu_{i}-1} E_{\rho_{i}, \mu_{i}}^{\gamma_{i}}\left[\omega_{i}\left(\psi_{i}\left(x_{i}\right)-\psi_{i}\left(t_{i}\right)\right)^{\rho_{i}}\right] d t_{i}\right)=
\end{gathered}
$$

(by 11), 12) )

$$
\prod_{i=1}^{N}\left[\left(\psi_{i}\left(x_{i}\right)-\psi_{i}\left(a_{i}\right)\right)^{\mu_{i}} E_{\rho_{i}, \mu_{i}+1}^{\gamma_{i}}\left[\omega_{i}\left(\psi_{i}\left(x_{i}\right)-\psi_{i}\left(a_{i}\right)\right)^{\rho_{i}}\right]\right] .
$$

That is

$$
K_{a+}(x)=\prod_{i=1}^{N}\left[\left(\psi_{i}\left(x_{i}\right)-\psi_{i}\left(a_{i}\right)\right)^{\mu_{i}} E_{\rho_{i}, \mu_{i}+1}^{\gamma_{i}}\left[\omega_{i}\left(\psi_{i}\left(x_{i}\right)-\psi_{i}\left(a_{i}\right)\right)^{\rho_{i}}\right]\right]
$$

$\forall x \in \prod_{i=1}^{N}\left(a_{i}, b_{i}\right)$.

Similarly, we compute the integral

$$
\begin{aligned}
& K_{b-}(x):=\int_{\prod_{i=1}^{N}\left(a_{i}, b_{i}\right)} k_{b-}(x, y) d y \stackrel{\text { 5) }}{=}\left({ }^{M} e_{\rho, \mu, \omega, b-}^{\gamma ; \psi} 1\right)(x)= \\
& \prod_{i=1}^{N}\left[\left(\psi_{i}\left(b_{i}\right)-\psi_{i}\left(x_{i}\right)\right)^{\mu_{i}} E_{\rho_{i}, \mu_{i}+1}^{\gamma_{i}}\left[\omega_{i}\left(\psi_{i}\left(b_{i}\right)-\psi_{i}\left(x_{i}\right)\right)^{\rho_{i}}\right]\right],
\end{aligned}
$$

$\forall x \in \prod_{i=1}^{N}\left(a_{i}, b_{i}\right)$.

Next, we form the ratios

$$
\begin{aligned}
& \frac{k_{a+}(x, y)}{K_{a+}(x)}= \\
& \frac{\chi_{\prod_{i=1}^{N}\left(a_{i}, x_{i}\right]}(y) \prod_{i=1}^{N}\left[\psi_{i}^{\prime}\left(y_{i}\right)\left(\psi_{i}\left(x_{i}\right)-\psi_{i}\left(y_{i}\right)\right)^{\mu_{i}-1} E_{\rho_{i}, \mu_{i}}^{\gamma_{i}}\left[\omega_{i}\left(\psi_{i}\left(x_{i}\right)-\psi_{i}\left(y_{i}\right)\right)^{\rho_{i}}\right]\right]}{\prod_{i=1}^{N}\left[\left(\psi_{i}\left(x_{i}\right)-\psi_{i}\left(a_{i}\right)\right)^{\mu_{i}} E_{\rho_{i}, \mu_{i}+1}^{\gamma_{i}}\left[\omega_{i}\left(\psi_{i}\left(x_{i}\right)-\psi_{i}\left(a_{i}\right)\right)^{\rho_{i}}\right]\right]}
\end{aligned}
$$




$$
\begin{gathered}
=\chi_{\prod_{i=1}^{N}\left(a_{i}, x_{i}\right]}(y) \prod_{i=1}^{N}\left(\frac{\psi_{i}^{\prime}\left(y_{i}\right)\left(\psi_{i}\left(x_{i}\right)-\psi_{i}\left(y_{i}\right)\right)^{\mu_{i}-1}}{\left(\psi_{i}\left(x_{i}\right)-\psi_{i}\left(a_{i}\right)\right)^{\mu_{i}}}\right) \\
\prod_{i=1}^{N}\left(\frac{E_{\rho_{i}, \mu_{i}}^{\gamma_{i}}\left[\omega_{i}\left(\psi_{i}\left(x_{i}\right)-\psi_{i}\left(y_{i}\right)\right)^{\rho_{i}}\right]}{E_{\rho_{i}, \mu_{i}+1}^{\gamma_{i}}\left[\omega_{i}\left(\psi_{i}\left(x_{i}\right)-\psi_{i}\left(a_{i}\right)\right)^{\rho_{i}}\right]}\right),
\end{gathered}
$$

and

$$
\begin{aligned}
\frac{k_{b-}(x, y)}{K_{b-}(x)}= & \chi_{\prod_{i=1}^{N}\left[x_{i}, b_{i}\right)}(y) \prod_{i=1}^{N}\left(\frac{\psi_{i}^{\prime}\left(y_{i}\right)\left(\psi_{i}\left(y_{i}\right)-\psi_{i}\left(x_{i}\right)\right)^{\mu_{i}-1}}{\left(\psi_{i}\left(b_{i}\right)-\psi_{i}\left(x_{i}\right)\right)^{\mu_{i}}}\right) \\
& \prod_{i=1}^{N}\left(\frac{E_{\rho_{i}, \mu_{i}}^{\gamma_{i}}\left[\omega_{i}\left(\psi_{i}\left(y_{i}\right)-\psi_{i}\left(x_{i}\right)\right)^{\rho_{i}}\right]}{E_{\rho_{i}, \mu_{i}+1}^{\gamma_{i}}\left[\omega_{i}\left(\psi_{i}\left(b_{i}\right)-\psi_{i}\left(x_{i}\right)\right)^{\rho_{i}}\right]}\right), \\
\forall x, y \in \prod_{i=1}^{N}\left(a_{i}, b_{i}\right) . &
\end{aligned}
$$

In this work we prove a variety of interesting generalized multivariate Hardy type fractional inequalities under convexity, related to (4) and (5), mixed generalized multivariate left and right Prabhakar type fractional integrals.

\section{Prerequisites}

I) Let $\left(\Omega_{1}, \Sigma_{1}, \mu_{1}\right)$ and $\left(\Omega_{2}, \Sigma_{2}, \mu_{2}\right)$ be measure spaces with positive $\sigma$-finite measures, and let $k_{j}: \Omega_{1} \times \Omega_{2} \rightarrow \mathbb{R}$ be nonnegative measurable functions, $k_{j}(x, \cdot)$ measurable on $\Omega_{2}$, and

$$
K_{j}(x)=\int_{\Omega_{2}} k_{j}(x, y) d \mu_{2}(y), \text { for any } x \in \Omega_{1},
$$

$j=1, \ldots, r \in \mathbb{N}$. We assume that $K_{j}(x)>0$ a.e. on $\Omega_{1}$ and the weight function are nonnegative measurable functions on the related set.

We consider measurable functions $g_{j}: \Omega_{1} \rightarrow \mathbb{R}$ with the representation

$$
g_{j}(x)=\int_{\Omega_{2}} k_{j}(x, y) f_{j}(y) d \mu_{2}(y),
$$

where $f_{j}: \Omega_{2} \rightarrow \mathbb{R}$ are measurable functions, $j=1, \ldots, r$. Here $u$ stands for a weight function on $\Omega_{1}$ (nonnegative measurable function).

We mention

Theorem 2.1. (1], p. 29) Let $\bar{j} \in\{1, \ldots, r\}$ be fixed. Assume that the function $x \rightarrow\left(\frac{u(x) \prod_{j=1}^{r} k_{j}(x, y)}{\prod_{j=1}^{r} K_{j}(x)}\right)$ is integrable on $\Omega_{1}$, for each $y \in \Omega_{2}$. Define $\lambda_{r}$ on $\Omega_{2}$ by

$$
\lambda_{r}(y):=\int_{\Omega_{1}}\left(\frac{u(x) \prod_{j=1}^{r} k_{j}(x, y)}{\prod_{j=1}^{r} K_{j}(x)}\right) d \mu_{1}(x)<\infty .
$$

Here $\Phi_{j}: \mathbb{R}_{+} \rightarrow \mathbb{R}_{+}, j=1, \ldots, r$, are convex and increasing functions.

Then

$$
\int_{\Omega_{1}} u(x) \prod_{j=1}^{r} \Phi_{j}\left(\left|\frac{g_{j}(x)}{K_{j}(x)}\right|\right) d \mu_{1}(x) \leq
$$


GENERALIZED MULTIVARIATE PRABHAKAR TYPE FRACTIONAL INTEGRALS AND INEQUALITIE\$

$$
\left(\prod_{\substack{j=1 \\ j \neq \bar{j}}}^{r} \int_{\Omega_{2}} \Phi_{j}\left(\left|f_{j}(y)\right|\right) d \mu_{2}(y)\right)\left(\int_{\Omega_{2}} \Phi_{\bar{j}}\left(\left|f_{\bar{j}}(y)\right|\right) \lambda_{r}(y) d \mu_{2}(y)\right),
$$

true for all measurable functions, $j=1, \ldots, r, f_{j}: \Omega_{2} \rightarrow \mathbb{R}$ such that:

(i) $f_{j}, \Omega_{j}\left(\left|f_{j}\right|\right)$, are both $k_{j}(x, y) d \mu_{2}(y)$ - integrable, $\mu_{1}$-a.e. in $x \in \Omega_{1}$,

(ii) $\left.\lambda_{r} \Phi_{\bar{j}}\left(\left|f_{\bar{j}}\right|\right) ; \Phi_{1}\left(\left|f_{1}\right|\right), \Phi_{2}\left(\left|f_{2}\right|\right), \Phi_{3}\left(\left|f_{3}\right|\right), \ldots, \overline{\Phi_{\bar{j}}\left(\left|f_{\bar{j}}\right|\right.}\right), \ldots, \Phi_{r}\left(\left|f_{r}\right|\right)$, are all $\mu_{2}$-integrable,

and for all corresponding functions $g_{j}$ given by $\widehat{21}$. Above $\overline{\Phi_{\bar{j}}\left(\left|f_{\bar{j}}\right|\right)}$ means missing item.

II) We need

Remark 2.2. In the next we consider for $j=1, \ldots, r$, the measurable functions $f_{1 j}, f_{2 j}: \Omega_{2} \rightarrow \mathbb{R}$, and

$$
g_{1 j}(x)=\int_{\Omega_{2}} k_{j}(x, y) f_{1 j}(y) d \mu_{2}(y)
$$

and

$$
g_{2 j}(x)=\int_{\Omega_{2}} k_{j}(x, y) f_{2 j}(y) d \mu_{2}(y),
$$

these are now instead of (21).

Again here $u \geq 0$ is a weight measurable function on $\Omega_{1}$.

We will use the following rational result:

Theorem 2.3. (2], p. 405) Here $0<f_{2 j}(y)<\infty$, a.e., $j=1, \ldots$, r. Let $\bar{j} \in$ $\{1, \ldots, r\}$ be fixed. Assume that the function $x \rightarrow\left(\frac{u(x) \prod_{j=1}^{r} k_{j}(x, y) f_{2 j}(y)}{\prod_{j=1}^{r} g_{2 j}(x)}\right)$ is integrable on $\Omega_{1}$, for each $y \in \Omega_{2}$. Define $\lambda_{r}^{* *}$ on $\Omega_{2}$ by

$$
\lambda_{r}^{* *}(y):=\left(\prod_{j=1}^{r} f_{2 j}(y)\right) \int_{\Omega_{1}}\left(\frac{u(x) \prod_{j=1}^{r} k_{j}(x, y)}{\prod_{j=1}^{r} g_{2 j}(x)}\right) d \mu_{1}(x)<\infty .
$$

Here $\Phi_{j}: \mathbb{R}_{+} \rightarrow \mathbb{R}_{+}, j=1, \ldots, r$, are convex and increasing functions.

Then

$$
\begin{gathered}
\int_{\Omega_{1}} u(x) \prod_{j=1}^{r} \Phi_{j}\left(\left|\frac{g_{1 j}(x)}{g_{2 j}(x)}\right|\right) d \mu_{1}(x) \leq \\
\left(\prod_{\substack{j=1 \\
j \neq \bar{j}}}^{r} \int_{\Omega_{2}} \Phi_{j}\left(\left|\frac{f_{1 j}(y)}{f_{2 j}(y)}\right|\right) d \mu_{2}(y)\right)\left(\int_{\Omega_{2}} \Phi_{\bar{j}}\left(\left|\frac{f_{1 \bar{j}}(y)}{f_{2 \bar{j}}(y)}\right|\right) \lambda_{r}^{* *}(y) d \mu_{2}(y)\right),
\end{gathered}
$$

true for all measurable functions, $j=1, \ldots, r, f_{1 j}, f_{2 j}: \Omega_{2} \rightarrow \mathbb{R}$ such that:

(i) $\frac{f_{1 j}(y)}{f_{2 j}(y)}, \Phi_{j}\left(\left|\frac{f_{1 j}(y)}{f_{2 j}(y)}\right|\right)$, are both $k_{j}(x, y) f_{2 j}(y) d \mu_{2}(y)$ - integrable, $\mu_{1}$-a.e. in $x \in \Omega_{1}$, 
(ii) $\lambda_{r}^{* *} \Phi_{\bar{j}}\left(\left|\frac{f_{1 \bar{j}}(y)}{f_{2 \bar{j}}(y)}\right|\right)$, and $\Phi_{j}\left(\left|\frac{f_{1 j}(y)}{f_{2 j}(y)}\right|\right)$, for $j=\{1, \ldots, r\}-\{\bar{j}\}$, are all $\mu_{2}-$ integrable;

and for all corresponding $g_{1 j}$ given by (24), and $g_{2 j}$ given by (25).

III) Here we follow [2] p. 441, see Chapter 22.

Let $\left(\Omega_{1}, \Sigma_{1}, \mu_{1}\right)$ and $\left(\Omega_{2}, \Sigma_{2}, \mu_{2}\right)$ be measure spaces with positive $\sigma$-finite measures, and let $k_{j}: \Omega_{1} \times \Omega_{2} \rightarrow \mathbb{R}$ be nonnegative measurable functions, $k_{j}(x, \cdot)$ measurable on $\Omega_{2}$, and

$$
K_{j}(x)=\int_{\Omega_{2}} k_{j}(x, y) d \mu_{2}(y), \text { for any } x \in \Omega_{1},
$$

$j=1, \ldots, r \in \mathbb{N}$. We assume that $K_{j}(x)>0$ a.e. on $\Omega_{1}$ and the weight functions are nonnegative measurable functions on the related set.

We consider measurable functions $g_{j}: \Omega_{1} \rightarrow \mathbb{R}$ with the representation

$$
g_{j}(x)=\int_{\Omega_{2}} k_{j}(x, y) f_{j}(y) d \mu_{2}(y),
$$

where $f_{j}: \Omega_{2} \rightarrow \mathbb{R}$ are measurable functions, $j=1, \ldots, r$.

Here $u$ stands for a weight function on $\Omega_{1}$ ( $u \geq 0$, which is measurable).

We will use the following general result:

Theorem 2.4. (2], p. 442) Assume that the functions $(j=1,2, \ldots, r \in \mathbb{N}) x \rightarrow$ $\left(u(x) \frac{k_{j}(x, y)}{K_{j}(x)}\right)$ are integrable on $\Omega_{1}$, for each fixed $y \in \Omega_{2}$. Define $u_{j}$ on $\Omega_{2}$ by

$$
u_{j}(y):=\int_{\Omega_{1}} u(x) \frac{k_{j}(x, y)}{K_{j}(x)} d \mu_{1}(x)<\infty .
$$

Let $p_{j}>1: \sum_{j=1}^{r} \frac{1}{p_{j}}=1$. Let the functions $\Phi_{j}: \mathbb{R}_{+} \rightarrow \mathbb{R}_{+}, j=1, \ldots, r$, be convex and increasing.

Then

$$
\begin{gathered}
\int_{\Omega_{1}} u(x) \prod_{j=1}^{r} \Phi j\left(\left|\frac{g_{j}(x)}{K_{j}(x)}\right|\right) d \mu_{1}(x) \leq \\
\prod_{j=1}^{r}\left(\int_{\Omega_{2}} u_{j}(y) \Phi_{j}\left(\left|f_{j}(y)\right|\right)^{p_{j}} d \mu_{2}(y)\right)^{\frac{1}{p_{j}}},
\end{gathered}
$$

for all measurable functions $f_{j}: \Omega_{2} \rightarrow \mathbb{R}(j=1, \ldots, r)$ such that

(i) $f_{j}, \Phi_{j}\left(\left|f_{j}\right|\right)^{p_{j}}$, are both $k_{j}(x, y) d \mu_{2}(y)$ - integrable, $\mu_{1}$-a.e. in $x \in \Omega_{1}$, $j=1, \ldots, r$,

(ii) $u_{j} \Phi_{j}\left(\left|f_{j}\right|\right)^{p_{j}}$ is $\mu_{2}$-integrable, $j=1, \ldots, r$,

and for all corresponding functions $g_{j}(j=1, \ldots, r)$ given by 29).

IV) Let $\left(\Omega_{1}, \Sigma_{1}, \mu_{1}\right)$ and $\left(\Omega_{2}, \Sigma_{2}, \mu_{2}\right)$ be measure spaces with positive $\sigma$-finite measures, and let $k: \Omega_{1} \times \Omega_{2} \rightarrow \mathbb{R}$ be nonnegative measurable functions, $k(x, \cdot)$ measurable on $\Omega_{2}$, and

$$
K(x)=\int_{\Omega_{2}} k(x, y) d \mu_{2}(y), \text { for any } x \in \Omega_{1} .
$$

We suppose that $K(x)>0$ a.e. on $\Omega_{1}$ and by a weight function $u$ (shortly: a weight), we mean a nonnegative measurable function on the actual set. Let the 
measurable functions $g_{j}: \Omega_{1} \rightarrow \mathbb{R}, j=1, \ldots, r$, with the representation

$$
g_{j}(x)=\int_{\Omega_{2}} k(x, y) f_{j}(y) d \mu_{2}(y),
$$

where $f_{j}: \Omega_{2} \rightarrow \mathbb{R}$ are measurable functions, $j=1, \ldots, r$.

Denote by $\vec{x}=x:=\left(x_{1}, \ldots, x_{r}\right) \in \mathbb{R}^{r}, \vec{g}:=\left(g_{1}, \ldots, g_{r}\right)$ and $\vec{f}:=\left(f_{1}, \ldots, f_{r}\right)$.

We consider here $\Phi: \mathbb{R}_{+}^{r} \rightarrow \mathbb{R}$ a convex function, which is increasing per coordinate, i.e. if $x_{j} \leq y_{j}, j=1, \ldots, r$, then

$$
\Phi\left(x_{1}, \ldots, x_{r}\right) \leq \Phi\left(y_{1}, \ldots, y_{r}\right) .
$$

In 2], p. 588, we proved that

Theorem 2.5. Let $u$ be a weight function on $\Omega_{1}$, and $k, K, g_{j}, f_{j}, j=1, \ldots, r \in \mathbb{N}$, and $\Phi$ defined as above. Assume that the function $x \rightarrow u(x) \frac{k(x, y)}{K(x)}$ is integrable on $\Omega_{1}$ for each fixed $y \in \Omega_{2}$. Define $v$ on $\Omega_{2}$ by

$$
v(y):=\int_{\Omega_{1}} u(x) \frac{k(x, y)}{K(x)} d \mu_{1}(x)<\infty .
$$

Then

$$
\begin{gathered}
\int_{\Omega_{1}} u(x) \Phi\left(\frac{\left|g_{1}(x)\right|}{K(x)}, \ldots, \frac{\left|g_{r}(x)\right|}{K(x)}\right) d \mu_{1}(x) \leq \\
\int_{\Omega_{2}} v(y) \Phi\left(\left|f_{1}(y)\right|, \ldots,\left|f_{r}(y)\right|\right) d \mu_{2}(y),
\end{gathered}
$$

under the assumptions:

(i) $f_{j}, \Phi\left(\left|f_{1}\right|, \ldots,\left|f_{r}\right|\right)$, are $k(x, y) d \mu_{2}(y)$-integrable, $\mu_{1}$-a.e. in $x \in \Omega_{1}$, for all $j=1, \ldots, r$,

(ii) $v(y) \Phi\left(\left|f_{1}(y)\right|, \ldots,\left|f_{r}(y)\right|\right)$ is $\mu_{2}$-integrable.

\section{Main Results}

From now on in this work we assume $\rho_{i}, \mu_{i}, \gamma_{i}, \omega_{i}>0$, for $i=1, \ldots, N$. Clearly here $\left({ }^{M} e_{\rho, \mu, \omega, a+}^{\gamma ; \psi} f\right),\left({ }^{M} e_{\rho, \mu, \omega, b-}^{\gamma ; \psi} f\right)$ are measurable functions over $\prod_{i=1}^{N}\left[a_{i}, b_{i}\right]$, where $f \in C\left(\prod_{i=1}^{N}\left[a_{i}, b_{i}\right]\right)$. Infact by Theorem 1.3 these are integrable functions on $\prod_{i=1}^{N}\left[a_{i}, b_{i}\right]$.

I') Here we apply Theorem 2.1 to $44, \Omega 5$ for $f_{j} \in C\left(\prod_{i=1}^{N}\left[a_{i}, b_{i}\right]\right), j=1, \ldots, r$.

Theorem 3.1. Let $\bar{j} \in\{1, \ldots, r\}$ be fixed. Assume that the function $x \rightarrow$ $\left(u(x)\left(\frac{k_{a+}(x, y)}{K_{a+}(x)}\right)^{r}\right)$ is integrable on $\prod_{i=1}^{N}\left(a_{i}, b_{i}\right)$, for each $y \in \prod_{i=1}^{N}\left(a_{i}, b_{i}\right)$, see 16), 18. Here $u \geq 0$ stands for a weight function on $\prod_{i=1}^{N}\left(a_{i}, b_{i}\right)$. Define $\lambda_{r}^{+}$on $\prod_{i=1}^{N}\left(a_{i}, b_{i}\right)$ by

$$
\lambda_{r}^{+}(y):=\int_{\prod_{i=1}^{N}\left(a_{i}, b_{i}\right)}\left(u(x)\left(\frac{k_{a+}(x, y)}{K_{a+}(x)}\right)^{r}\right) d x<\infty,
$$


which is assumed to be integrable on $\prod_{i=1}^{N}\left(a_{i}, b_{i}\right)$.

Here $\Phi_{j}: \mathbb{R}_{+} \rightarrow \mathbb{R}_{+}, j=1, \ldots, r$, are convex and increasing functions.

Then

$$
\begin{gathered}
\int_{\prod_{i=1}^{N}\left(a_{i}, b_{i}\right)} u(x) \prod_{j=1}^{r} \Phi_{j}\left(\frac{\left|\left({ }^{M} e_{\rho, \mu, \omega, a+}^{\gamma ; \psi} f_{j}\right)(x)\right|}{\prod_{i=1}^{N}\left[\left(\psi_{i}\left(x_{i}\right)-\psi_{i}\left(a_{i}\right)\right)^{\mu_{i}} E_{\rho_{i}, \mu_{i}+1}^{\gamma_{i}}\left[\omega_{i}\left(\psi_{i}\left(x_{i}\right)-\psi_{i}\left(a_{i}\right)\right)^{\rho_{i}}\right]\right]}\right) d x \\
\leq\left(\prod_{\substack{j=1 \\
j \neq \bar{j}}}^{r} \int_{\prod_{i=1}^{N}\left(a_{i}, b_{i}\right)} \Phi_{j}\left(\left|f_{j}(y)\right|\right) d y\right)\left(\int_{\prod_{i=1}^{N}\left(a_{i}, b_{i}\right)} \Phi_{\bar{j}}\left(\left|f_{\bar{j}}(y)\right|\right) \lambda_{r}^{+}(y) d y\right) .
\end{gathered}
$$

We continue with

Theorem 3.2. Let $\bar{j} \in\{1, \ldots, r\}$ be fixed. Assume that the function $x \rightarrow$ $\left(u(x)\left(\frac{k_{b-}(x, y)}{K_{b-}(x)}\right)^{r}\right)$ is integrable on $\prod_{i=1}^{N}\left(a_{i}, b_{i}\right)$, for each $y \in \prod_{i=1}^{N}\left(a_{i}, b_{i}\right)$, see 1 1 $)$, 19.). Here $u \geq 0$ stands for a weight function on $\prod_{i=1}^{N}\left(a_{i}, b_{i}\right)$. Define $\lambda_{r}^{-}$on $\prod_{i=1}^{N}\left(a_{i}, b_{i}\right)$ by

$$
\lambda_{r}^{-}(y):=\int_{\prod_{i=1}^{N}\left(a_{i}, b_{i}\right)}\left(u(x)\left(\frac{k_{b-}(x, y)}{K_{b-}(x)}\right)^{r}\right) d x<\infty,
$$

which is assumed to be integrable on $\prod_{i=1}^{N}\left(a_{i}, b_{i}\right)$.

Here $\Phi_{j}: \mathbb{R}_{+} \rightarrow \mathbb{R}_{+}, j=1, \ldots, r$, are convex and increasing functions.

Then

$$
\begin{gathered}
\int_{\prod_{i=1}^{N}\left(a_{i}, b_{i}\right)} u(x) \prod_{j=1}^{r} \Phi_{j}\left(\frac{\left|\left({ }^{M} e_{\rho, \mu, \omega, b-}^{\gamma ; \psi} f_{j}\right)(x)\right|}{\prod_{i=1}^{N}\left[\left(\psi_{i}\left(b_{i}\right)-\psi_{i}\left(x_{i}\right)\right)^{\mu_{i}} E_{\rho_{i}, \mu_{i}+1}^{\gamma_{i}}\left[\omega_{i}\left(\psi_{i}\left(b_{i}\right)-\psi_{i}\left(x_{i}\right)\right)^{\rho_{i}}\right]\right]}\right) d x \\
\leq\left(\prod_{\substack{j=1 \\
j \neq \bar{j}}}^{r} \int_{\prod_{i=1}^{N}\left(a_{i}, b_{i}\right)} \Phi_{j}\left(\left|f_{j}(y)\right|\right) d y\right)\left(\int_{\prod_{i=1}^{N}\left(a_{i}, b_{i}\right)} \Phi_{\bar{j}}\left(\left|f_{\bar{j}}(y)\right|\right) \lambda_{r}^{-}(y) d y\right) .
\end{gathered}
$$

II') Here we apply Theorem 2.3 to 44,5 for $f_{1 j}, f_{2 j} \in C\left(\prod_{i=1}^{N}\left[a_{i}, b_{i}\right]\right), j=$ $1, \ldots, r$.

Theorem 3.3. Consider $f_{2 j}>0, j=1, \ldots, r$. Let $\bar{j} \in\{1, \ldots, r\}$ be fixed. Assume that the function $x \rightarrow\left(u(x)\left(k_{a+}(x, y)\right)^{r} \prod_{j=1}^{r}\left(\frac{f_{2 j}(y)}{\left({ }^{M} e_{\rho, \psi, \omega, a+}^{\gamma, \psi} f_{2 j}\right)(x)}\right)\right)$ (see 16 16$)$ is 
GENERALIZED MULTIVARIATE PRABHAKAR TYPE FRACTIONAL INTEGRALS AND INEQUALITIE\$ integrable on $\prod_{i=1}^{N}\left(a_{i}, b_{i}\right)$, for each $y \in \prod_{i=1}^{N}\left(a_{i}, b_{i}\right)$. Define $\lambda_{r}^{*+}$ on $\prod_{i=1}^{N}\left(a_{i}, b_{i}\right)$ by

$$
\lambda_{r}^{*+}(y):=\left(\prod_{j=1}^{r} f_{2 j}(y)\right) \int_{\prod_{i=1}^{N}\left(a_{i}, b_{i}\right)}\left(\frac{u(x)\left(k_{a+}(x, y)\right)^{r}}{\prod_{j=1}^{r}\left(M e_{\rho, \mu, \omega, a+}^{\gamma ; \psi} f_{2 j}(x)\right)}\right) d x<\infty
$$

for appropiate weight $u \geq 0$, so that $\lambda_{r}^{*+}$ is integrable on $\prod_{i=1}^{N}\left(a_{i}, b_{i}\right)$.

Here $\Phi_{j}: \mathbb{R}_{+} \rightarrow \mathbb{R}_{+}, j=1, \ldots, r$, are convex and increasing functions. Then

$$
\begin{gathered}
\int_{\prod_{i=1}^{N}\left(a_{i}, b_{i}\right)} u(x) \prod_{j=1}^{r} \Phi_{j}\left(\frac{\left|{ }^{M} e_{\rho, \mu, \omega, a+}^{\gamma ; \psi} f_{1 j}(x)\right|}{M e_{\rho, \mu, \omega, a+}^{\gamma ; \psi} f_{2 j}(x)}\right) d x \leq \\
\left(\prod_{\substack{j=1 \\
j \neq \bar{j}}}^{r} \int_{\prod_{i=1}^{N}\left(a_{i}, b_{i}\right)} \Phi_{j}\left(\frac{\left|f_{1 j}(y)\right|}{f_{2 j}(y)}\right) d y\right)\left(\int_{\prod_{i=1}^{N}\left(a_{i}, b_{i}\right)} \Phi_{\bar{j}}\left(\frac{\left|f_{1 \bar{j}}(y)\right|}{f_{2 \bar{j}}(y)}\right) \lambda_{r}^{*+}(y) d y\right) .
\end{gathered}
$$

We continue with

Theorem 3.4. Consider $f_{2 j}>0, j=1, \ldots, r$. Let $\bar{j} \in\{1, \ldots, r\}$ be fixed. Assume that the function $x \rightarrow\left(u(x)\left(k_{b-}(x, y)\right)^{r} \prod_{j=1}^{r}\left(\frac{f_{2 j}(y)}{\left(M e_{\rho, \mu, \omega, b-}^{\gamma ; \psi} f_{2 j}\right)(x)}\right)\right)$ (see 1 1 1 ) is integrable on $\prod_{i=1}^{N}\left(a_{i}, b_{i}\right)$, for each $y \in \prod_{i=1}^{N}\left(a_{i}, b_{i}\right)$. Define $\lambda_{r}^{*-}$ on $\prod_{i=1}^{N}\left(a_{i}, b_{i}\right)$ by

$$
\lambda_{r}^{*-}(y):=\left(\prod_{j=1}^{r} f_{2 j}(y)\right) \int_{\prod_{i=1}^{N}\left(a_{i}, b_{i}\right)}\left(\frac{u(x)\left(k_{b-}(x, y)\right)^{r}}{\prod_{j=1}^{r}\left(M e_{\rho, \mu, \omega, b-}^{\gamma ; \psi} f_{2 j}(x)\right)}\right) d x<\infty,
$$

for appropiate weight $u \geq 0$, so that $\lambda_{r}^{*-}$ is integrable on $\prod_{i=1}^{N}\left(a_{i}, b_{i}\right)$.

Here $\Phi_{j}: \mathbb{R}_{+} \rightarrow \mathbb{R}_{+}, j=1, \ldots, r$, are convex and increasing functions. Then

$$
\begin{gathered}
\int_{\prod_{i=1}^{N}\left(a_{i}, b_{i}\right)} u(x) \prod_{j=1}^{r} \Phi_{j}\left(\frac{\left|{ }^{M} e_{\rho, \mu, \omega, b-}^{\gamma ; \psi} f_{1 j}(x)\right|}{M e_{\rho, \mu, \omega, b-}^{\gamma ; \psi} f_{2 j}(x)}\right) d x \leq \\
\left(\prod_{\substack{j=1 \\
j \neq \bar{j}}}^{r} \int_{\prod_{i=1}^{N}\left(a_{i}, b_{i}\right)} \Phi_{j}\left(\frac{\left|f_{1 j}(y)\right|}{f_{2 j}(y)}\right) d y\right)\left(\int_{\prod_{i=1}^{N}\left(a_{i}, b_{i}\right)} \Phi_{\bar{j}}\left(\frac{\left|f_{1 \bar{j}}(y)\right|}{f_{2 \bar{j}}(y)}\right) \lambda_{r}^{*-}(y) d y\right) .
\end{gathered}
$$

III') Here we apply Theorem 2.4 to $44,\left\{5\right.$ for $f_{j} \in C\left(\prod_{i=1}^{N}\left[a_{i}, b_{i}\right]\right), j=1, \ldots, r$. 
Theorem 3.5. Assume that the function $x \rightarrow\left(u(x) \frac{k_{a+}(x, y)}{K_{a+}(x)}\right)$ is integrable on $\prod_{i=1}^{N}\left(a_{i}, b_{i}\right)$, for each fixed $y \in \prod_{i=1}^{N}\left(a_{i}, b_{i}\right)$. Define $\rho^{+}$on $\prod_{i=1}^{N}\left(a_{i}, b_{i}\right)$ by

$$
\rho^{+}(y):=\int_{\prod_{i=1}^{N}\left(a_{i}, b_{i}\right)} u(x) \frac{k_{a+}(x, y)}{K_{a+}(x)} d x<\infty,
$$

(see (13), 16), 18)) for appropiate weight $u \geq 0$, so that $\rho^{+}$is integrable on $\prod_{i=1}^{N}\left(a_{i}, b_{i}\right)$.

Let $p_{j}>1: \sum_{j=1}^{r} \frac{1}{p_{j}}=1$. Let the functions $\Phi_{j}: \mathbb{R}_{+} \rightarrow \mathbb{R}_{+}, j=1, \ldots, r$, be convex and increasing.

Then

$$
\begin{gathered}
\int_{\prod_{i=1}^{N}\left(a_{i}, b_{i}\right)} u(x) \prod_{j=1}^{r} \Phi_{j}\left(\frac{\left|M e_{\rho, \mu, \omega, a+}^{\gamma ; \psi} f_{j}(x)\right|}{K_{a+}(x)}\right) d x \leq \\
\prod_{j=1}^{r}\left(\int_{\prod_{i=1}^{N}\left(a_{i}, b_{i}\right)} \rho^{+}(y) \Phi_{j}\left(\left|f_{j}(y)\right|\right)^{p_{j}} d y\right)^{\frac{1}{p_{j}}} .
\end{gathered}
$$

We continue with

Theorem 3.6. Assume that the function $x \rightarrow\left(u(x) \frac{k_{b-}(x, y)}{K_{b-}(x)}\right)$ is integrable on $\prod_{i=1}^{N}\left(a_{i}, b_{i}\right)$, for each fixed $y \in \prod_{i=1}^{N}\left(a_{i}, b_{i}\right)$. Define $\rho^{-}$on $\prod_{i=1}^{N}\left(a_{i}, b_{i}\right)$ by

$$
\rho^{-}(y):=\int_{\prod_{i=1}^{N}\left(a_{i}, b_{i}\right)} u(x) \frac{k_{b-}(x, y)}{K_{b-}(x)} d x<\infty,
$$

(see (14), 17), 199) for appropiate weight $u \geq 0$, so that $\rho^{-}$is integrable on $\prod_{i=1}^{N}\left(a_{i}, b_{i}\right)$.

Let $p_{j}>1: \sum_{j=1}^{r} \frac{1}{p_{j}}=1$. Let the functions $\Phi_{j}: \mathbb{R}_{+} \rightarrow \mathbb{R}_{+}, j=1, \ldots, r$, be convex and increasing.

Then

$$
\begin{gathered}
\int_{\prod_{i=1}^{N}\left(a_{i}, b_{i}\right)} u(x) \prod_{j=1}^{r} \Phi_{j}\left(\frac{\left|M e_{\rho, \mu, \omega, b-}^{\gamma ; \psi} f_{j}(x)\right|}{K_{b-}(x)}\right) d x \leq \\
\prod_{j=1}^{r}\left(\int_{\prod_{i=1}^{N}\left(a_{i}, b_{i}\right)} \rho^{-}(y) \Phi_{j}\left(\left|f_{j}(y)\right|\right)^{p_{j}} d y\right)^{\frac{1}{p_{j}}} .
\end{gathered}
$$

IV') Here we apply Theorem 2.5 to $44, \quad 5$ for $f_{j} \in C\left(\prod_{i=1}^{N}\left[a_{i}, b_{i}\right]\right), j=1, \ldots, r$. 
Theorem 3.7. Consider here $\Phi: \mathbb{R}_{+}^{r} \rightarrow \mathbb{R}$ a convex function, which is increasing per coordinate, and $u \geq 0$ the weight. Assume that the function $x \rightarrow u(x) \frac{k_{a+}(x, y)}{K_{a+}(x)}$ is integrable on $\prod_{i=1}^{N}\left(a_{i}, b_{i}\right)$ for each $y \in \prod_{i=1}^{N}\left(a_{i}, b_{i}\right)$. Define $\nu^{+}$on $\prod_{i=1}^{N}\left(a_{i}, b_{i}\right)$ (see (16), (18)) by

$$
\nu^{+}(y):=\int_{\prod_{i=1}^{N}\left(a_{i}, b_{i}\right)} u(x) \frac{k_{a+}(x, y)}{K_{a+}(x)} d x<\infty,
$$

for appropiate $u$, so that $\nu^{+}$is integrable on $\prod_{i=1}^{N}\left(a_{i}, b_{i}\right)$. Then

$$
\begin{gathered}
\int_{\prod_{i=1}^{N}\left(a_{i}, b_{i}\right)} u(x) \Phi\left(\frac{\left|{ }^{M} e_{\rho, \mu, \omega, a+}^{\gamma ; \psi} f_{1}(x)\right|}{K_{a+}(x)}, \ldots, \frac{\left|{ }^{M} e_{\rho, \mu, \omega, a+}^{\gamma ; \psi} f_{r}(x)\right|}{K_{a+}(x)}\right) d x \leq \\
\int_{\prod_{i=1}^{N}\left(a_{i}, b_{i}\right)} \nu^{+}(y) \Phi\left(\left|f_{1}(y)\right|, \ldots,\left|f_{r}(y)\right|\right) d y .
\end{gathered}
$$

We also give

Theorem 3.8. Consider here $\Phi: \mathbb{R}_{+}^{r} \rightarrow \mathbb{R}$ a convex function, which is increasing per coordinate, and $u \geq 0$ the weight. Assume that the function $x \rightarrow u(x) \frac{k_{b-}(x, y)}{K_{b-}(x)}$ is integrable on $\prod_{i=1}^{N}\left(a_{i}, b_{i}\right)$ for each $y \in \prod_{i=1}^{N}\left(a_{i}, b_{i}\right)$. Define $\nu^{-}$on $\prod_{i=1}^{N}\left(a_{i}, b_{i}\right)$ (see (17), (19)) by

$$
\nu^{-}(y):=\int_{\prod_{i=1}^{N}\left(a_{i}, b_{i}\right)} u(x) \frac{k_{b-}(x, y)}{K_{b-}(x)} d x<\infty,
$$

for appropiate $u$, so that $\nu^{-}$is integrable on $\prod_{i=1}^{N}\left(a_{i}, b_{i}\right)$. Then

$$
\begin{gathered}
\int_{\prod_{i=1}^{N}\left(a_{i}, b_{i}\right)} u(x) \Phi\left(\frac{\left|{ }^{M} e_{\rho, \mu, \omega, b-}^{\gamma ; \psi} f_{1}(x)\right|}{K_{b-}(x)}, \ldots, \frac{\left|{ }^{M} e_{\rho, \mu, \omega, b-}^{\gamma ; \psi} f_{r}(x)\right|}{K_{b-}(x)}\right) d x \leq \\
\int_{\prod_{i=1}^{N}\left(a_{i}, b_{i}\right)} \nu^{-}(y) \Phi\left(\left|f_{1}(y)\right|, \ldots,\left|f_{r}(y)\right|\right) d y .
\end{gathered}
$$

One can produce a vast wealth of similar results, related to (4) and (5), by applying the general results of [2], Chapters 21-27, but we choose here to skip this task.

We mention

Definition 3.9. All as in $(4)$ and $(5), x \in \prod_{i=1}^{N}\left[a_{i}, b_{i}\right]$. We define the left and right partial Prabhakar fractional integrals with respect to $\psi_{k}$, respectively, for $k=$ $1, \ldots, N$, as follows:

$$
\begin{gathered}
\left({ }^{P} e_{\rho_{k}, \mu_{k}, \omega_{k}, a_{k}+}^{\gamma_{k} ; \psi_{k}} f\right)(x):=\int_{a_{k}}^{x_{k}} \psi_{k}^{\prime}\left(t_{k}\right)\left(\psi_{k}\left(x_{k}\right)-\psi_{k}\left(t_{k}\right)\right)^{\mu_{k}-1} \\
\left.E_{\rho_{k}, \mu_{k}}^{\gamma_{k}}\left[\omega_{k}\left(\psi_{k}\left(x_{k}\right)-\psi_{k}\left(t_{k}\right)\right)^{\rho_{k}}\right]\right] f\left(x_{1}, \ldots, x_{k-1}, t_{k}, x_{k+1}, \ldots, x_{N}\right) d t_{k},
\end{gathered}
$$


with $a_{k} \leq x_{k} \leq b_{k}$;

and

$$
\begin{gathered}
\left({ }^{P} e_{\rho_{k}, \mu_{k}, \omega_{k}, b_{k}-}^{\gamma_{k} ; \psi_{k}} f\right)(x):=\int_{x_{k}}^{b_{k}} \psi_{k}^{\prime}\left(t_{k}\right)\left(\psi_{k}\left(t_{k}\right)-\psi_{k}\left(x_{k}\right)\right)^{\mu_{k}-1} \\
\left.E_{\rho_{k}, \mu_{k}}^{\gamma_{k}}\left[\omega_{k}\left(\psi_{k}\left(t_{k}\right)-\psi_{k}\left(x_{k}\right)\right)^{\rho_{k}}\right]\right] f\left(x_{1}, \ldots, x_{k-1}, t_{k}, x_{k+1}, \ldots, x_{N}\right) d t_{k},
\end{gathered}
$$

with $a_{k} \leq x_{k} \leq b_{k}$.

By [3], 52), 53) are continuous functions in $x_{k}, k=1, \ldots, N$.

We need

Definition 3.10. ([16], p. 35) Here we consider the Prabhakar function with respect to another function $\alpha(x)>0, x \in[a, b], \alpha \in C([a, b])$, as follows:

$$
E_{\alpha(x), \beta}^{\gamma}(z):=\sum_{k=0}^{\infty} \frac{(\gamma)_{k}}{k ! \Gamma(k \alpha(x)+\beta)} z^{k}
$$

where $\beta>0, \gamma \in \mathbb{R}, z \in \mathbb{R}$.

We mention

Definition 3.11. All as in (4) and (5), however now instead of $\rho$ we have $\rho(x)=$ $\left(\rho_{1}\left(x_{1}\right), \ldots, \rho_{N}\left(x_{N}\right)\right)$, where $0<\rho_{i} \in C\left(\left[a_{i}, b_{i}\right]\right)$, for $i=1, \ldots, N$. We define the left and right mixed Prabhakar multiple fractional integrals of variable degree with respect to $\psi$, respectively, as follows:

$$
\begin{gathered}
\left({ }_{v}^{M} e_{\rho, \mu, \omega, a+}^{\gamma ; \psi} f\right)(x):=\int_{a_{1}}^{x_{1}} \ldots \int_{a_{N}}^{x_{N}} \prod_{i=1}^{N}\left[\psi_{i}^{\prime}\left(t_{i}\right)\left(\psi_{i}\left(x_{i}\right)-\psi_{i}\left(t_{i}\right)\right)^{\mu_{i}-1}\right. \\
\left.E_{\rho_{i}\left(t_{i}\right), \mu_{i}}^{\gamma_{i}}\left[\omega_{i}\left(\psi_{i}\left(x_{i}\right)-\psi_{i}\left(t_{i}\right)\right)^{\rho_{i}\left(t_{i}\right)}\right]\right] f\left(t_{1}, \ldots, t_{N}\right) d t_{1} \ldots d t_{N},
\end{gathered}
$$

with $x_{i} \geq a_{i}, i=1, \ldots, N$;

$$
\begin{gathered}
\left({ }_{v}^{M} e_{\rho, \mu, \omega, b-}^{\gamma ; \psi} f\right)(x):=\int_{x_{1}}^{b_{1}} \ldots \int_{x_{N}}^{b_{N}} \prod_{i=1}^{N}\left[\psi_{i}^{\prime}\left(t_{i}\right)\left(\psi_{i}\left(t_{i}\right)-\psi_{i}\left(x_{i}\right)\right)^{\mu_{i}-1}\right. \\
\left.E_{\rho_{i}\left(t_{i}\right), \mu_{i}}^{\gamma_{i}}\left[\omega_{i}\left(\psi_{i}\left(t_{i}\right)-\psi_{i}\left(x_{i}\right)\right)^{\rho_{i}\left(t_{i}\right)}\right]\right] f\left(t_{1}, \ldots, t_{N}\right) d t_{1} \ldots d t_{N},
\end{gathered}
$$

with $x_{i} \leq b_{i}, i=1, \ldots, N$.

One can prove similar results as above and in [2], Chapters 21-27, for the operators $(52),(53), 55),(56)$, but we omit here this task.

\section{REFERENCES}

[1] G.A. Anastassiou, Univariate Hardy-type fractional inequalities, Chapter 2, in "Advances in Applied Mathematics and Approximation Theory", Contributions from AMAT 2012, G. Anastassiou, O. Duman Editors, Springer, New York, 2013, pp. 21-56.

[2] G.A. Anastassiou, Intelligent Comparisons: Analytic Inequalities, Springer, Heidelberg, New York, 2016.

[3] G.A. Anastassiou, Foundations of Generalized Prabhakar-Hilfer fractional Calculus with Applications, submitted, 2021.

[4] A. Giusti et al, A practical Guide to Prabhakar Fractional Calculus, Fractional Calculus \& Applied Analysis, Vol. 23 (1) (2020), 9-54.

[5] R. Gorenflo, A. Kilbas, F. Mainardi, S. Rogosin, Mittag-Leffler functions, Related Topics and Applications, Springer, Heidelberg, New York, 2014.

[6] H.G. Hardy, Notes on some points in the integral calculus, Messenger of Mathematics, vol. 47 , no. $10,1918,145-150$. 
[7] E. Hewith, K. Stromberg, Real and Abstract Analysis, Springer, New York, Heidelberg, Berlin, 1965.

[8] S. Iqbal, K. Krulic and J. Pecaric, On an inequality of H.G. Hardy, J. of Inequalities and Applications, Volume 2010, Article ID 264347, 23 pages.

[9] S. Iqbal, K. Krulic, J. Pecaric, On an inequality for convex functions with some applications on fractional derivatives and fractional integrals, Journal of Mathematical Inequalities, Vol. 5, No. 2 (2011), 219-230.

[10] A.A. Kilbas, M. Saigo, R.K. Saxena, Generalized Mittag-Leffler function and generalized fractional calculus operators, Integral Transforms and special Functions, 15 (1) (2004), 3149.

[11] F. Polito, Z. Tomovski, Some properties of Prabhakar-type fractional calculus operators, Fractional Differential Calculus, Vol. 6 (1), (2016), 73-94.

[12] T.R. Prabhakar, A singular integral equation with a generalized Mittag Leffler function in the kernel, Yokohama Math. J., 19 (1971), 7-15.

[13] R.K. Saxena, S.L. Kalla, R. Saxena, Multivariate analogue of generalized Mittag-Leffler function, Integral Transforms and special Functions, 22 (7) (2011), 533-548.

[14] H.M. Srivastava, Z. Tomovski, Fractional calculus with an integral operator containing a generalized Mittag-Leffler function in the kernel, Applied Mathematics and Computation, 211 (2009), 198-210.

[15] Ž. Tomovski, R. Hilfer, H.M. Srivastava, Fractional and Operational Calculus with Generalized Fractional Derivative Operators and Mittag-Leffler Type Functions, Integral Transforms Spec Funct., 21 (11) (2010), 797-814.

[16] X.-J. Yang, general Fractional Derivatives. Theory, Methods and Applications, CRC Press, Taylor \& Francis Group, Boca Raton, FL, 2019.

George A. Anastassiou

Department of Mathematical Sciences, University of Memphis, Memphis, TN 38152, U.S.A.

E-mail address: ganastss@memphis.edu 